prescribers working remotely or on call can now complete Drug Instruction Charts in a timely way, improving experience for patients, families and staff.

\section{P-143 DEVELOPMENT OF PALLIATIVE CARE SAFE PRESCRIBING RESOURCES FOR PRIMARY CARE}

Karen Menzies. Ayrshire Hospice, Ayr, UK

\subsection{6/spcare-2021-Hospice. 160}

Background Anecdotal evidence from community pharmacies (CPs) and care homes indicate palliative care medicines are often incorrectly prescribed due to lack of knowledge of CD (controlled drugs) regulations and of doses/formulations used. These incidents did not lead to patient harm but changes to prescriptions cause unnecessary delays to symptom management for patients and additional stress for carers collecting medicines.

Aims The aims of the project were to produce resources for primary care teams to help support safe prescribing and supply of these medicines and to raise awareness of the Community Pharmacy Palliative Care Network. The network pharmacies keep an agreed stock list of palliative medicines and receive annual generalist palliative care training. We also wanted to challenge CPs' awareness of the issues families face when trying to access palliative medicines and support them to improve their service.

Methods

- Baseline surveys to gain feedback from community pharmacy staff, GP practice staff and district nurses (DN) on issues with prescribing and supply of palliative care medicines.

- Developed resources with key safe prescribing messages and distributed to all CPs and GP clinical pharmacists, GP practices, district nurses and care homes, with copies of the Scottish Palliative Care Guideline handbooks and Palliative Care Network leaflet.

- Presented to local conferences and team meetings about project and distributed resources.

- Improve awareness of 40 Palliative Care Network Pharmacies and how they can help support palliative patients.

Outcomes

- The baseline survey showed 78\% of survey respondents were aware of the Palliative Care Network Pharmacies, repeated survey showed this had increased to $92 \%$.

- 'What influences your choice of community pharmacy?'- the response, 'Good relationship with community pharmacy' increased from $18 \%$ to $52 \%$.

- Repeated requests for more resources.

\section{P-144 THE ROLE OF NON-MEDICAL PRESCRIBERS}

Anne-Marie O'Connell. Keech Hospice Care, Luton, UK

10.1136/spcare-2021-Hospice.161

Within our hospice we have a team of four senior nurses working as Non-Medical Prescribers (NMP). The role was being developed prior to the pandemic, however, was initiated during lockdown when some of the medical team were shielding. With the doctors now fully available the aim is still to bridge the gaps in medical cover such as extended hours over the weekends and to facilitate annual leave/training or cover sickness.

We also work to support the nursing team for training, medication queries and as a mediator for discussions with the medical team, where needed. We work to assist with discharge planning, external referrals, gathering information and equipment loans. We work to support all areas of the hospice as part of the multi-disciplinary team including adult inpatient unit, wellbeing centre, out-of-hours advice line, accepting referrals at weekends and palliative care support workers.

Moving forward the hope is to expand the nurse-led clinics that are supported by the NMP role. We currently run a 'Looking forward' clinic for patients with liver disease and plan to offer similar clinics for cardiac and respiratory diseases. As we are able to open up our services further we plan to reinstate access to the Wellbeing Centre for patient assessments such as complex symptom management and discharge follow ups.

As advanced practitioners we have an awareness of our own limitations, and each of us has our own experience and expertise from previous roles, which brings value to the team as a whole. It is still a developing role and we are one of the few hospices to have a NMP team in post. With good communication, a broad skill mix, regular sharing of opinions and ongoing education this helps us to be an effective service for supporting all areas of the hospice.

\section{P-145 POST FALLS MANAGEMENT}

Debbie Pegram, Hannah Chapman, Anne Bisset-Smith, Alexander Palomo. St Joseph's Hospice, London, UK

10.1136/spcare-2021-Hospice. 162

Background We became aware, following an audit, that there were a number of patient falls in which the post falls assessment would have benefited from being more comprehensive. There is a risk that if someone is moved without being fully assessed for potential fractures or neurological damage, they may sustain further injury. Therefore, we embarked on a quality improvement project, with the aim of improving our post falls assessment.

$\operatorname{Aim}(s)$

- For all staff to be aware of the correct procedure to follow after a patient falls.

- To increase staff knowledge around their role in post fall management.

- Reduce the risk of any harm.

Methods

- The post falls assessment process was laid out using NICE guidelines.

- Baseline measures were taken:

- Doctors and nurses were given three scenarios and asked a set of questions

- Documentation of previous falls was audited.

- Post Falls Pathway was developed, introduced and placed in each room.

- Bite size education was delivered.

- Baseline measure was repeated, with the documentation of falls following the introduction of the pathway being audited. 
Results The scenario scores increased from an average of $81.75 \%$ to $96.75 \%$. The audit scores increased from $63 \%$ to $100 \%$.

Conclusions A combination of the comprehensive guidance, including the Post Falls Pathway and staff education (including how to safely manoeuvre a patient after a fall), has demonstrated an increase in confidence and consistency of assessment and has reduced the risk of harm. Our approach has demonstrated that the introduction of the pathway has significantly improved the safe management of patients who have experienced a fall.

\section{P-146 OUR QUALITY IMPROVEMENT JOURNEY AT HOSPICE ISLE OF MAN: SMALL CHANGES NEED SMALL STEPS}

Tracy Broom, David Waters, Cheryl Young, Ben Harris, Anne Mills. Hospice Isle of Man, Douglas, Isle of Man

\subsection{6/spcare-2021-Hospice.163}

Background In June 2021, the hospice began delivering our first ever Quality Improvement (QI) Programme. We had observed an environment that was innovative in research and could be innovative in QI. We all 'improved quality' but did we use QI as a framework for that improvement?

Aims Our shared vision was to integrate QI into the normal working day, within a culture of continuous improvement (National Advisory Group on the Safety of Patients in England, 2013). We have now launched our QI journey with our first sequential and small steps; a QI Programme and QI Champions.

Methods The programme was delivered over four days to clinical and non-clinical staff; motivated and energetic people from every department in Hospice. We delivered QI technical skills (Institute for Healthcare Improvement. How to improve [The Model for Improvement]) blending theory and practice, whilst also focusing on wider topics which support QI landing successfully in the day job. This included improvement leadership (Øvretveit, 2009), human factors (National Quality Board, 2013) and team work (Montgomery, Parkin, Chisholm, et al., 2020). These specific topics were chosen by the participants, so the programme was bespoke to their learning needs and overall the programme was designed to be a fun and interactive environment.

Results We commenced evaluation with a pre-course selfassessment of QI knowledge, skills and confidence. This was repeated at the end of each facilitated day and will be concluded by a post-course self-assessment. The first cohort completes in September 2021 and we will have the programme evaluation completed the same month.

Conclusions We anticipate participants will have improved QI knowledge, skills and confidence which will enable them to actively use QI in their normal working day and will also transition to become QI Champions. The QI Champion is a new role being designed to support other staff, encourage momentum and be a key part of building a network of QI Champions. We would like to share our story so far and future system plans. Sharing, learning, improving.

\section{P-147 ABSTRACT WITHDRAWN}

\section{P-148 INPATIENTS HOSPICE ADMISSIONS, WHO IS ADMITTED AND WHY: A MIXED METHOD PROSPECTIVE STUDY}

1,2Erna Haraldsdottir, ${ }^{3}$ Libby Milton, ${ }^{1}$ Anna Lloyd, ${ }^{3,4}$ Anne Finucane, ${ }^{3}$ Martyn Bijak, ${ }^{1} J a c k i e$ Stone, ${ }^{1}$ Dot Partington, ${ }^{3}$ Hilary Ford, 'Duncan Brown. 'St Columba's Hospice Care, Edinburgh, UK; ${ }^{2}$ Queen Margaret University, Edinburgh, UK; ${ }^{3}$ Marie Curie Hospice, Edinburgh, UK; ${ }^{4}$ University of Edinburgh, Edinburgh, UK

\subsection{6/spcare-2021-Hospice. 164}

Background Across the UK, more people are projected to die in community settings over the next two decades. The role of the hospice inpatient unit (IPU) needs to be better understood in light of these shifts. The term complex needs is used for patients admitted to IPU, however, there is little clarity around these needs and how they trigger admission.

Aim To understand why patients are admitted to IPU and to describe the palliative care services available to them beforehand.

Method Prospective mixed methods study. Data were collected in two hospices in one city of Scotland. We examined case notes of 259 patients admitted to the IPU over four months and conducted 40 semi-structured interviews. (22 patients or a relative proxy, 11 health care professionals, 7 pro-forma interviews-source of referrals).

Results Mean age was 71 years, 53\% were female; 47\% male. Most patients admitted were Scottish or White British (95\%). Most were living with another person at the time of admission (72\%); 28\% lived alone. The vast majority had cancer (95\%). Phase of illness was judged as deteriorating or unstable for over two-thirds at the time of admission. Most patients were receiving specialist palliative care support prior to admission $-73 \%$ had a community palliative care CNS (Clinical Nurse Specialist). Just under half had district nurse support (48\%). Over one-fifth (21\%) had no prior community palliative care involvement; most of these patients were referred from the hospital (81\%). Length of stay was 12 days (median). 68\% of admissions were for end-of-life care, with the patient dying during the admission. The hospice inpatient unit was the documented preferred place of death for $56 \%$ who died in that location.

Thematic analysis of the qualitative data identified the key reasons for admissions, symptom control, anxiety and fear, social isolation and end-of-life care.

\section{Conclusion}

- Admission to inpatient hospice is a last resort and often a response to a crisis situation.

- Greater palliative care support in home settings is needed so that more patients can remain at home when that is their preference.

\section{P-149 A REVIEW OF OUTCOME MEASURES USED BY SPEECH AND LANGUAGE THERAPISTS IN ADULTS UNDER PALLIATIVE CARE}

${ }^{1}$ Charlotte Robinson, ${ }^{2}$ Eli Harriss, ${ }^{1}$ Mary Miller. ' Oxford University Hospitals NHS Foundation Trust, Oxford, UK; ${ }^{2}$ Bodleian Library, Oxford University, Oxford, UK

\subsection{6/spcare-2021-Hospice. 165}

Introduction Little is known about the use of outcome measures $(\mathrm{OM})$ by Speech and Language Therapists (SLTs) in palliative care. This review aimed to identify studies using validated $\mathrm{OMs}$ in adults receiving palliative care, and to 